were lower in LA/CFA, though not statistically significant from AdA. Lesions developed more slowly in LA/CFA than in AdA. However, hock BMD as well as knee inflammation and erosion scores found to be different (shown here at Onset +5 days):

Conclusion Inflammatory and skeletal changes for AdA and LA/ CFA were similar by clinical and histopathologic measures 10 days after onset, while inflammation receded by Onset +25 days in LA/CFA more than it did in AdA. These data indicate that bone densitometric and morphologic findings can distinguish these two models of adjuvant arthritis despite their similar clinical presentations. This finding may be relevant in screening novel therapeutic candidates as these models may define different potential efficacious effects of a compound.

\section{OP0093 INTERFERON GAMMA GENE POLYMORPHISM: RELATIONSHIP TO SUSCEPTIBILITY AND SEVERITY OF RHEUMATOID ARTHRITIS IN A PROSPECTIVE STUDY}

\begin{abstract}
${ }^{1,2,3} \mathrm{~A}$ Constantin, ${ }^{2} \mathrm{~F}$ Navaux, ${ }^{1} \mathrm{~V}$ Lauwers-Cancès, ${ }^{3} \mathrm{M}$ Abbal, ${ }^{4} \mathrm{JP}$ Van Meerjwijk, ${ }^{2} \mathrm{~B}$ Mazières, ${ }^{1} \mathrm{~A}$ Cambon-Thomsen, ${ }^{1,3} \mathrm{~A}$ Cantagrel. ${ }^{1}$ Rheumatology Service, Rangueil Hospital, Toulouse, France; ${ }^{2}$ INSERM, Unité 558, Toulouse, France; ${ }^{3}$ Immunology Service, Rangueil Hospital, INSERM Unité 395, Toulouse, France
\end{abstract}

\subsection{6/annrheumdis-2001.100}

Background Interferon gamma (IFNg) is a multifunctional cytokine that plays an important role in modulating most of the phases of the immune and inflammatory responses. IFNg gene is a potential candidate as marker of rheumatoid arthritis (RA) susceptibility and severity.

Objectives In this prospective longitudinal study we have investigated the association between a variable length CA repeat in the first intron of the IFNg gene and RA susceptibility and severity.

Methods One hundred and three early ( $<1$ year) RA patients (ACR 1987 criteria) were evaluated clinically, serologically and radiographically (modified Sharp/van der Heijde method) yearly for 4 years. The total radiological damage score was used to quantify RA severity after 4 years of follow-up. One hundred thirty unrelated healthy individuals were used as controls. The microsatellite region in the first intron of the IFNg gene was analysed by PCR and electrophoresis in an automatic DNA sequencer.

Results Characteristics of the 103 RA patients at inclusion were as follows: 80 females, 23 males, mean age $49.4 \pm 15.5$ years, mean disease duration $6.9 \pm 3.7$ months, mean tender joint score $12.4 \pm 8.9$, mean swollen joint number $6 \pm 6.2$, mean erythrocyte sedimentation rate $29.8 \pm 25.2$, rheumatoid factor positive (ELISA method) 71/103, mean radiological score $5 \pm$ 13.6. The allele sizes of the IFNg gene polymorphic region ranged from $122 \mathrm{bp}$ (11 CA repeats) to $134 \mathrm{bp}$ (17 CA repeats). Twelve CA repeat (12R) and thirteen CA repeat (13R) alleles were the most common in RA patients (respectively $41.7 \%$ and $48.5 \%$ ) and healthy controls (respectively $45.3 \%$ and $45.7 \%$ ). $12 \mathrm{R} / 12 \mathrm{R}, 12 \mathrm{R} / 13 \mathrm{R}$ and $13 \mathrm{R} / 13 \mathrm{R}$ genotypes were the most common in RA patients (respectively $15.6 \%, 44.1 \%$ and $21.6 \%$ ) and healthy controls (respectively 22.4\%, 38.0\% and 21.7\%). No association has been observed between the IFNg gene polymorphism and RA susceptibility or severity.

Conclusion Although the present study fails to reveal any association between this particular IFNg gene polymorphism and RA, the search for RA susceptibility and severity genes remains an important issue to better understand RA pathophysiology and to optimise the strategies for treatment of early RA.

\section{OP0096 TRANSCRIPTOME ANALYSIS IN RHEUMATOID ARTHRITIS TREATED WITH A TNF NEUTRALISING TNF RECEPTOR: FUSION PROTEIN (ETANERCEPT)}

${ }^{1} \mathrm{~J}$ Kekow, ${ }^{1} \mathrm{~S}$ Drynda, ${ }^{2} \mathrm{D}$ Koczan, ${ }^{2} \mathrm{HJ}$ Thiesen. ${ }^{1} \mathrm{Clinic}$ of Rheumatology, Otto-Von-GuerickeUniversity, Magdeburg; ${ }^{2}$ Institut for Immunology, University of Rostock, Rostock, Germany

\subsection{6/annrheumdis-2001.101}

Background Treatment with etanercept (Enbrel) results in a rapid and sustained improvement of disease activity in patients with refractory rheumatoid arthritis (RA). Although changes in the expression of single proteins, particularly cytokines and proteases, could be demonstrated in the past at the protein and mRNA level, new methods are neccessary to provide insight into the complex mechanisms which occur after neutralisation of TNF $\alpha$.

Objectives Oligonucleotide arrays can provide a broad picture of the state of cells by monitoring the expression levels of thousands of genes at the same time. Here we report the application of DNA array technology (Affymetrix) to cover changes in the expression levels under etanercept treatment.

Methods Mononuclear cells (PBMC) and neutrophils (PMN) from peripheral blood were prepared applying standard procedures, synovial fluid (SF) was obtained from both knees. Total RNA for analysis was isolated from PBMC, PMN and SF-cells at different time points in the course of treatment.

Results The expression level of 5600 transcripts was analysed. The most expressed genes were found in SF-cells. About 3.3\%, $2.4 \%$ and $0.2 \%$ of the assessed genes in PBMC, PMN and SFcells, respectively, showed significant changes in gene expression in either direction (i.e. increase with a fold change $>/=3$, decrease with a fold change.

Conclusion The group of genes which are up- or downregulated included a number of genes which are known to be involved in transcription, chemotaxis, cell adhesion as well as genes not previously examined in the context of rheumatoid arthritis.

The results have to be considered preliminary and confirmed in further experiments.

\section{OP0097 JUVENILE IDIOPATHIC ARTHRITIS (JIA) IS ASSOCIATED WITH THE D6S265 MICROSATELLITE POLYMORPHISMS ON THE HIGH-RISK DR8-DQ4 HAPLOTYPE}

${ }^{1} \mathrm{~A}$ Smerdel, ${ }^{2} \mathrm{BA}$ Lie, ${ }^{3} \mathrm{R}$ Ploski, ${ }^{2} \mathrm{DE}$ Undlien, ${ }^{4} \mathrm{~B}$ Flato, ${ }^{2} \mathrm{E}$ Thorsby, ${ }^{4} \mathrm{O}$ Forre. ${ }^{1}$ Institute of Immunology (On Leave of Absence From Institute of Rheumatology, Warsaw, Poland); ${ }^{2}$ Institute of Immunology; ${ }^{3}$ Institute of Rheumatology, Warsaw, Poland; ${ }^{4}$ Center for Rheumatic Diseases, The National Hospital, Oslo, Norway

\subsection{6/annrheumdis-2001.102}

Background Juvenile idiopathic arthritis (JIA) has been shown to be associated with at least four different alleles: HLA-A2, DR5 and DR6, and DP2. These associations are independent from each other, i.e. they are not caused by linkage disequilibrium between the markers. However, several studies have suggested that additional, unmapped, HLA-linked genes may participate in JIA.

Objectives The purpose of this study was to look for additional genes involved in the susceptibility for JIA in the HLA complex using microsatellite polymorphisms. Since it is known that JIA is strongly associated with the DR8-DQ4 haplotype only DR8DQ4 haplotype was included in the study, in order to exclude associations secondary to this high-risk haplotype. 
Methods We investigated $102, \mathrm{DRB} 1 * 08$ positive, patients with JIA classified according to American Collage of Rheumatology criteria. As controls we selected 270, DRB1*08 positive, healthy individuals. We scanned $12 \mathrm{Mb}$ of DNA covering the HLA complex using the following microsatellite polymorphisms, from centromeric to telomeric: D6S291, DQCar, D6S273, MIB, D6S265, D6S2223 and D6S2239. An EM algorythm was used to estimate haplotypes and only the distributions of microsatellite alleles on DR8-DQ4 haplotypes was compared between patients and controls.

Results The microsatellite locus D6S265, which is located centromeric of HLA-A, showed a strong positive association with the disease for the ${ }^{*} 130 \mathrm{bp}$ allele $\left(\mathrm{OR}=30, \mathrm{p}<1^{-6}\right)$. In order to investigate whether this association could be explained by HLA-A2 the frequency of the HLA-A2 allele on DR8-DQ4D6S265*130 haplotype was compared among patients and controls. However, the D6S265*130 allele still showed evidence for disease association $\left(\mathrm{OR}=23, \mathrm{pp}<1^{-5}\right)$.

Conclusion The observed association of D6S265*130 allele with JIA is not secondary to the association of this allele with the DR8-DQ4-A2 haplotype. It shows that D6S265*130 allele could be a marker for additional predisposing factor involved in JIA, and HLA-A2 cannot explain all associations to the JIA in HLA class I region.

\section{OP0098 THE JAK3 PROMOTER REGION IS STIMULATED BY T CELL ACTIVATION AND CONTAINS ESSENTIAL AP-1 AND ETS-1 ELEMENTS}

${ }^{1} \mathrm{M}$ Aringer, ${ }^{2} \mathrm{DM}$ Frucht, ${ }^{2} \mathrm{M}$ Chen, ${ }^{2} \mathrm{M}$ Centola, ${ }^{2} \mathrm{JJ}$ O'Shea. ${ }^{1}$ Rheumatology/Internal Medicine III, University of Vienna, Austria, Wien, Austria; ${ }^{2}$ Arthritis and Rheumatism Branch, NIAMS/National Institutes of Health, Bethesda, MD, USA

10.1136/annrheumdis-2001.103

Background Janus Kinase 3 (Jak3) is a non-receptor tyrosine kinase essential for interleukin- (IL-) 2, 4, 7, 9, and 15 signalling. In $\mathrm{T}$ lymphocytes, Jak3 expressed is clearly increased upon $\mathrm{T}$ cell activation. The regulatory regions of the Jak3 promoter, however, are unknown.

Objectives We therefore decided to characterise the promoter region of Jak3.

Methods A 5'race procedure was used to detect the beginning of Jak3 cDNA derived from both the Jurkat $\mathrm{T}$ cell line and an NK cell line (NK3.3). All sequencing was performed on an ABI 377 automated sequencer using DNA amplified by PCR and the ABI Big Dye terminator kit. Putative promoter pieces were amplified by PCR and subcloned into a luciferase vector. In addition, site-directed mutagenesis was used to change two AP-1 sites and a putative Stat binding site within the core promoter region. Jurkat T cells were transfected both with these constructs and a beta-galactosidase vector for normalisation; both activities were sequentially read on a luminometer using the Tropix Dual Light kit.

Results All Jak3 mRNA started 5' of a 3515 basepair intron; the first basepair of the longest mRNA species found was denominated as +1 . The 1039 basepair DNA region 5 of the mRNA start $(-1013 /+27)$ worked as a (TATA-less) promoter and could be activated by PMA, PHA, or immobilised anti-CD3, but not anti-CD28. As expected, the promoter did not work in the reverse direction. Truncation constructs showed the active region situated within a 267 basepair $(-240 /+27)$ region, the core promoter was positioned between ?74 and ?16. Within this region, that contained also an SP-1 site, a double-AP1 site, but not the potential Stat site in between, was of major importance, as demonstrated by targeted point mutation. Surrounding the core promoter, two Ets-1 consensus elements had significant (around 6fold) enhancer activity.

Conclusion The Jak3 promoter is situated around 3500 basepairs 5 of its ATG-codon. The promoter can be activated by T cell receptor crosslinking and contains an active double AP-1 binding site and Ets- 1 consensus elements that work as significant enhancers. This may lead to improved IL-2 (or ?4, -7- or ? 15) signalling in disease states where $T$ lymphocytes are being activated.

\section{THU0001 TNF-ALPHA POLYMORPHISM AT POSITION -308 MIGHT PROTECT AGAINST RADIOGRAPHIC DAMAGE IN RA IRRESPECTIVE OF RHEUMATOID FACTOR OR HLA STATUS}

${ }^{1} \mathrm{C}$ Orellana, ${ }^{1} \mathrm{R}$ Sanmartí, ${ }^{1} \mathrm{JD}$ Cañete, ${ }^{2} \mathrm{~J}$ Yagüe, ${ }^{2} \mathrm{G}$ Ercilla, ${ }^{1} \mathrm{~A}$ Gómez, ${ }^{1} \mathrm{G}$ Salvador, 1J Muñoz-Gómez. 'Department of Rheumatology; ${ }^{2}$ Department of Immunology, Hospital Clinic, Barcelona, Spain

10.1136/annrheumdis-2001.104

\section{Background}

Objectives To assess the prevalence of TNF-alpha polymorphisms in patients with early rheumatoid arthritis (RA) in our population and investigate whether there is an association with radiographic severity.

Methods 68 patients with early RA (49F/19M, mean age $51 \pm$ $15 \mathrm{y}$, mean disease duration $15 \pm 13 \mathrm{mo}, \mathrm{RF}+72 \%$, mean number of tender joints at entry $6.3 \pm 5.7$, mean number of swollen joints at entry $8.23 \pm 7.33$ ). Patients were genotyped for HLADRB1 and TNF-alpha alleles. Hands and feet radiographs were taken at entry and then yearly during follow-up. The Larsen method was used to assess radiographic damage.

Results There were not differences in the distribution of TNFalpha alleles in the RA patients compared with controls. The TNF-alpha -308GG genotype instead of the ?308GA genotype was found to be more frequent in patients with no erosive disease at entry compared with those presenting erosions $(89 \%$ vs $62 \%, \mathrm{p}=0.0023)$. Patients not showing radiographic erosions during follow-up $(36.5 \pm 22 \mathrm{mo})$ also had more frequently the $308 \mathrm{GG}$ genotype (96\% vs. $74 \%$ in erosive disease, $\mathrm{p}=0.018$ ). There were no differences in disease duration nor follow-up between patients with erosive and non erosive disease. The TNF-alpha -308GG polymorphism was as frequent in patients with non erosive disease as in controls, and there were not differences either among RF+ve and RF-ve patients nor among patients with or without the shared epitope or who were HLADR4 positive. No other associations related to TNF-alpha were found in relation to radiographic damage.

Conclusion The presence of the ?308GG TNF-alpha genotype appears to be one of the factors related to less severe disease as determined by erosive disease, and may have a protective role compared with the ?308 GA genotype. 